\title{
AGE AND SECONDARY TASK ENGAGEMENT IN RELATION TO SAFE/UNSAFE DRIVING BEHAVIOR AND CRASH/NON-CRASH EVENTS
}

\author{
Jose A. Calvo IV ${ }^{1}$, Carryl L. Baldwin ${ }^{1}$, \& Brian H. Philips ${ }^{2}$ \\ ${ }^{1}$ George Mason University, Fairfax, VA, USA \\ ${ }^{2}$ Federal Highway Administration Office of Safety R\&D, Washington, DC, USA \\ Email: jcalvo3@masonlive.gmu.edu
}

\begin{abstract}
Summary: Driver distraction is thought to play a causal role in automobile crashes. Younger and older drivers have the highest crash risk per mile driven. To get a better understanding of the risk associated with conducting secondary tasks while driving the Naturalistic Driving Study (NDS) dataset, part of the Second Strategic Highway Research Program (SHRP2) was used to run a log-linear model comparing age and secondary task involvement and their relation to Event Severity (Balanced Baseline vs Crash), as well as Maneuver Judgement (Safe vs Unsafe). A significant relationship was found between event severity and maneuver judgement. Additionally, age group and secondary task engagement had a significant effect on event severity, but no significant interaction between age and secondary task was found. Age had a significant effect on maneuver judgement, but secondary task did not. Therefore, maneuver judgement may not be a good substitute for event severity as an outcome variable for predicting crashes.
\end{abstract}

\section{INTRODUCTION}

Injuries and fatalities caused by motor vehicle crashes are a persistent threat to public safety (NHTSA, 2018). Distracted driving is a causal factor in many fatal and nonfatal crashes. The National Highway Traffic and Safety Administration (NHTSA) reported that in 2016 9\% (3157) of all fatal crashes were a result of distracted driving, resulting in a total of 3450 fatalities. Additionally, in $201515 \%$ or 265,000 non-fatal crashes were a result of distracted driving, $8 \%$ of which involved the use of cell phones. NHTSA has indicated that crashes resulting from distracted driving go largely under reported suggesting that distracted driving may be a greater concern than previously thought. There are several potential reasons for distracted driving to go under reported as a cause for crashes, especially fatal crashes. People typically shy away from self-reporting negative behavior, especially behavior that could have legal ramifications. Law enforcement officials do not have a universal method for writing up accident reports, sometimes there is not even an option for the officer to mark the crash as being caused by distraction. Finally, distraction can be hard to quantify and it may not always be clear to the reporting officer when distraction was the root of the crash (NHTSA, 2018). With this in consideration it is plausible that the rate of crashes due to distraction is even higher than what is currently being reported.

Distracted driving involves conducting or engaging in any task not associated with the task(s) needed to drive safely. The most common and widely cited form of distracted driving is the use or interaction with a cell phone and/or a geolocation positioning system (GPS) (Young \& Reagan 2007). However, according to NHTSA, in 2016 only 14\% of all reported distraction based fatal crashes involved cell phones. Furthermore, only $8 \%$ of non-fatal distraction-affected crashes involved cell phone use. There are many ways to classify or group types of secondary tasks. 
Secondary tasks can be categorized in terms of inputs, location, complexity, or by type of distraction (Metz, Landu, \& Just 2014; Owens et al. 2015). Not all secondary tasks are created equal, but distraction across all categories negatively impacts driving performance. Therefore, understanding how overall distraction affects driving performance across other factors that influence crashes is important.

Younger drivers, ages 16 to 24, and older drivers, ages 65 and above, have the highest crash rate per mile driven. Specifically, for younger drivers, drivers ages 15 to 19 make up $6 \%$ of all drivers and $9 \%$ of distracted drivers involved in fatal crashes in 2016, while drivers in their 20's make up $24 \%$ of all drivers, $28 \%$ of distracted drivers, and $32 \%$ of cell phone related distracted drivers involved in fatal crashes in 2016 (NHTSA 2018). These data would suggest a difference between newly licensed teen drivers and drivers in their 20s. Klauer et al. (2014) compared distracted driving and risk of road crashes for newly licensed drivers, ages 16 to 17, and experienced drivers, ages 18 to 72. Klauer et al.'s (2014) definition of experienced and inexperienced drivers would suggest that within the "younger driver" population there exists two groups. Recently licensed drivers ranging in age 16 to 19 would be young novice drivers (novice drivers), and drivers ages 20 to 24 who have been licensed for at least a year, would be experienced younger drivers (young drivers).

Guo et al. (2017) used the SHRP2 NDS data to compare four age groups across several levels of secondary task engagement and found that both age and distraction had a significant effect on the likelihood of a crash. Specifically, drivers in their 20 s were almost three times as likely to be involved in a crash while distracted followed closely by novice drivers and older drivers who are roughly two times as likely to be involved in a crash while distracted. These are compared to middle aged drivers who are only 1.5 times more likely to be involved in a crash while distracted. However, Guo et al. (2017) did not consider whether the driving maneuver made by the driver was safe or unsafe. While crash prediction is important, a lot more can be gained from understanding the relationship between distraction and safe/unsafe maneuvers. More importantly, older drivers may be at even greater risk of making unsafe maneuvers while engaged in secondary tasks, relative to younger drivers. Potentially younger drivers having more experience than older drivers at conducting these tasks may be able to engage in them more safely (allowing them to continue to make safe maneuvers) while age-related physical and cognitive changes among older adults may lead to secondary task engagement resulting in more unsafe maneuvers.

It is hypothesized that there will be a significant relationship between maneuver judgement and driving event, specifically that maneuvers judged to be unsafe will be more greatly associated with crashes compared to baselines. Furthermore, if these two do have a significant relationship, then it is possible that maneuver judgment can be used as an outcome variable instead of event severity for smaller naturalistic datasets that don't have a large enough sample of crashes to use as an outcome variable. It has been observed that both age and distraction have a significant effect on event severity. Therefore, is hypothesized that the effect of age and secondary task engagement will have a similar significant effect on maneuver judgement. To test this hypothesis the current study examined the best fit log-linear models for both event severity and maneuver judgement using age and secondary task engagement as predictors. 


\section{METHODS}

\section{Naturalistic Driving Study (NDS)}

Data from the SHRP2 NDS was used for this study. Data collection procedures and participant information can be found in the technical report by Dingus TA, et al. (2015) and the task report by Hankey, McClafferty, and Perez (2016).

\section{Data coding and study design}

The raw NDS data were aggregated so that events containing two or more crashes weren't counted multiple times. Only crashes that included physical contact with another object but with minimal damage, such as most road departures, small animal strikes, all curb and tires strikes potentially in conflict with oncoming traffic, and other curb strikes with an increased risk element (e.g., would have resulted in worse had curb not been there, usually related to driver behavior or state) were included. Within the NDS, balanced baselines, totaling 20,000 were taken from each participant based on time driven and were matched to driving patterns that were similar to patterns of driving that took place before a crash. Additionally, for all baselines and pre-crash maneuvers, the engagement of up to three secondary tasks were recorded and coded. For this study only the first of these secondary tasks was monitored. The initial 57 categories, which were identified for different secondary tasks, were aggregated into a binary response of either secondary task or no secondary task. The NDS dataset contained a variable termed "maneuver judgement", which was the judgement of a vehicles traversal of the roadway during a sample baseline epoch or the maneuver that took place just before a crash independent of the consideration of a driver's behavior. The maneuver could have been judged as safe and legal, safe but illegal, unsafe and illegal, unsafe but legal, or unknown. Unknown maneuver judgements were extremely infrequent and were removed from this analysis. Additionally, the other four levels were aggregated down to maneuvers being safe or unsafe regardless of their legality.

Following basic descriptive analyses, Chi squares were used to check for a significant relationship between maneuver judgement (pre-event maneuver or maneuver during a baseline judged to be either safe or unsafe) and event severity (baseline or crash). Additionally, loglinear models were used to analyze the relationships between age, secondary task engagement, and event severity, as well as age, secondary task engagement, and maneuver judgement.

\section{RESULTS}

\section{Relationships between factors}

Secondary task engagement differed as a function of age. Nearly $60 \%$ of drivers under the age of 25 were engaged in secondary tasks relative to just over $40 \%$ of those 65 and older. There was a significant relationship between maneuver judgement and event severity $X^{2}(1, N=16511)=$ $1193.4, p<.0001$ with more unsafe maneuvers being associated with crashes than expected if the two factors were independent of each other. This supports the hypothesis that maneuver judgement could potentially be used in place of event severity when trying to predict crash risk from naturalistic driving research.

\section{Loglinear Models}

A hierarchical model comparison was done to find the best fitting model for the relationship between age, secondary task engagement, and event severity. The breakdown for this model can 
be seen in Table 1. The model that best explained the relationship between age group, secondary task engagement, and event severity was the all two-way associations model. In other words, there was a significant main effect for both age and secondary task on event severity, but there was no significant interaction between age and secondary task on event severity. Figure 1 shows the comparison of the mutual independence model (expected results if there was no association between variables) to the observed results. Red shaded boxes suggest a lower likelihood for that specific combination of variables, while blue shaded boxes suggest a higher likelihood for that combination of variables. Furthermore, the intensity of the color indicates the strength of the likelihood for that outcome, dark red indicates a much lower likelihood than light red and dark blue indicates a much greater likelihood than light blue. For example, Figure 1 shows that for novice drivers engaged in a secondary task there is a higher likelihood of a crash scenario compared to a no crash scenario (balanced-baseline). Additionally, for a novice driver not engaged in a secondary task there is still a stronger likelihood of a cash scenario compared to a no crash scenario. However, both crash and no crash scenarios for when a novice driver is not engaged in a secondary task have a lower likelihood than crash and no crash scenarios for a novice driver engaged in a secondary task. For older drivers who are not engaged in secondary task there is a much higher likelihood that there would be no crash compared to a crash, and for older drivers engaged in a secondary task there is a slightly greater chance for them to be involved in a crash compared to a no crash scenario. For young drivers, there is a higher likelihood of them being engaged in a secondary task compared to no secondary task. Also, there is no difference between the likelihood of crash and no crash scenarios for young drivers engaged in a secondary task nor for younger drivers not engaged in a secondary task. Finally Middle age drivers both engaged in a secondary task and not engaged in a secondary task have a lower likelihood of being in a crash scenario compared to a no crash scenario, and there is no difference between their likelihood to engage or not to engage in secondary tasks.

A second hierarchical model comparison was done for maneuver judgment. The breakdown for this model can be seen in Table 2. The model that best explained the relationship between age group, secondary task engagement, and event severity was a conditional independence model. Specifically, it was found that age group significantly influenced maneuver judgement, but that when holding age group constant secondary task engagement did not influence maneuver judgement.

\section{CONCLUSIONS}

The current results indicate that when age is held constant, there was not a significant likelihood that secondary task engagement predicts safe or unsafe maneuvers. This is surprising since distraction is thought to play a significant causal role in crashes. One explanation for the current result could be that it isn't always dangerous to engage in secondary tasks while driving. Driving task demands are variable and shift as the environment, condition of roadway, and driving task requirements change. This means that under certain conditions secondary task engagement may be extremely difficult and can lead to a crash, but under different conditions that same secondary task can be performed quite easily while driving (Lansdown, Brook-Carter, Kersloot, \& Watt 2004; Young \& Regan 2007). Furthermore, the association between maneuver judgement and event severity could be influenced solely by age group and not by other factors such as distraction. The models for event severity and maneuver judgement were not similar enough to suggest that maneuver judgement could be used as a surrogate for event severity in other 
naturalistic driving datasets. However, it is possible that including maneuver judgement, breaking down secondary task by type, and adding in random effects could lead to the development of a stronger model and the understanding of which factors and how strongly those factors predict crashes.

Even though older adults are less likely to engage in secondary tasks, their crash rate per mile driven remains high, roughly the same as young drivers. This underscores previous results indicating that something other than voluntary behavior plays a causal role in the crashes of older drivers, while voluntary (e.g., risk-taking) behaviors play more of a role in the crashes of younger drivers.

\section{FIGURES AND TABLES}

Table 1. Hierarchical log-linear model comparison for the relationship of age, distraction, and event severity

\begin{tabular}{lccccc}
\hline \multicolumn{1}{c}{ Model Type } & Deviance & df & Delta(Dev) & Delta(df) & P(> Delta(Dev) \\
\hline Mutual Independence & 593.7504 & 10 & 0 & 0 & 0 \\
& & & & & \\
Joint Independence & 215.3864 & 7 & 378.364 & 3 & 0 \\
Conditional Independence (Secondary Task) & 136.7058 & 6 & 78.68059 & 1 & 0 \\
Conditional Independence (Age) & 67.8701 & 4 & 68.83572 & 2 & $1.11 \mathrm{E}-15$ \\
Homogeneous Independence & $\mathbf{6 . 2 2 3 3 1 1}$ & $\mathbf{3}$ & $\mathbf{6 1 . 6 4 6 7 9}$ & $\mathbf{1}$ & $\mathbf{4 . 1 1 E - 1 5}$ \\
Saturated & 0 & 0 & 6.223311 & 3 & 0.101237 \\
Observed & 0 & 0 & 0 & 0 & 1 \\
\hline
\end{tabular}

Table 2. Hierarchical log-linear model comparison of relationship between age, distraction and maneuver judgement

\begin{tabular}{lccccc}
\hline \multicolumn{1}{c}{ Model Type } & Deviance & df & Delta(Dev) & Delta(df) & P(> Delta(Dev) \\
\hline Mutual Independence & 491.4897518 & 10 & 0 & 0 & 0 \\
Joint Independence & 113.1257553 & 7 & 378.364 & 3 & 0 \\
Conditional Independence (Secondary Task) & 112.7841932 & 6 & 0.341562 & 1 & 0.558929 \\
Conditional Independence (Age) & $\mathbf{1 . 7 4 7 0 0 4 2 4 5}$ & $\mathbf{4}$ & $\mathbf{1 1 1 . 0 3 7 2}$ & $\mathbf{2}$ & $\mathbf{0}$ \\
Homogeneous Independence & 1.030987512 & 3 & 0.716017 & 1 & 0.397454 \\
Saturated & 0 & 0 & 1.030988 & 3 & 0.793754 \\
Observed & & & & & 1 \\
\hline
\end{tabular}




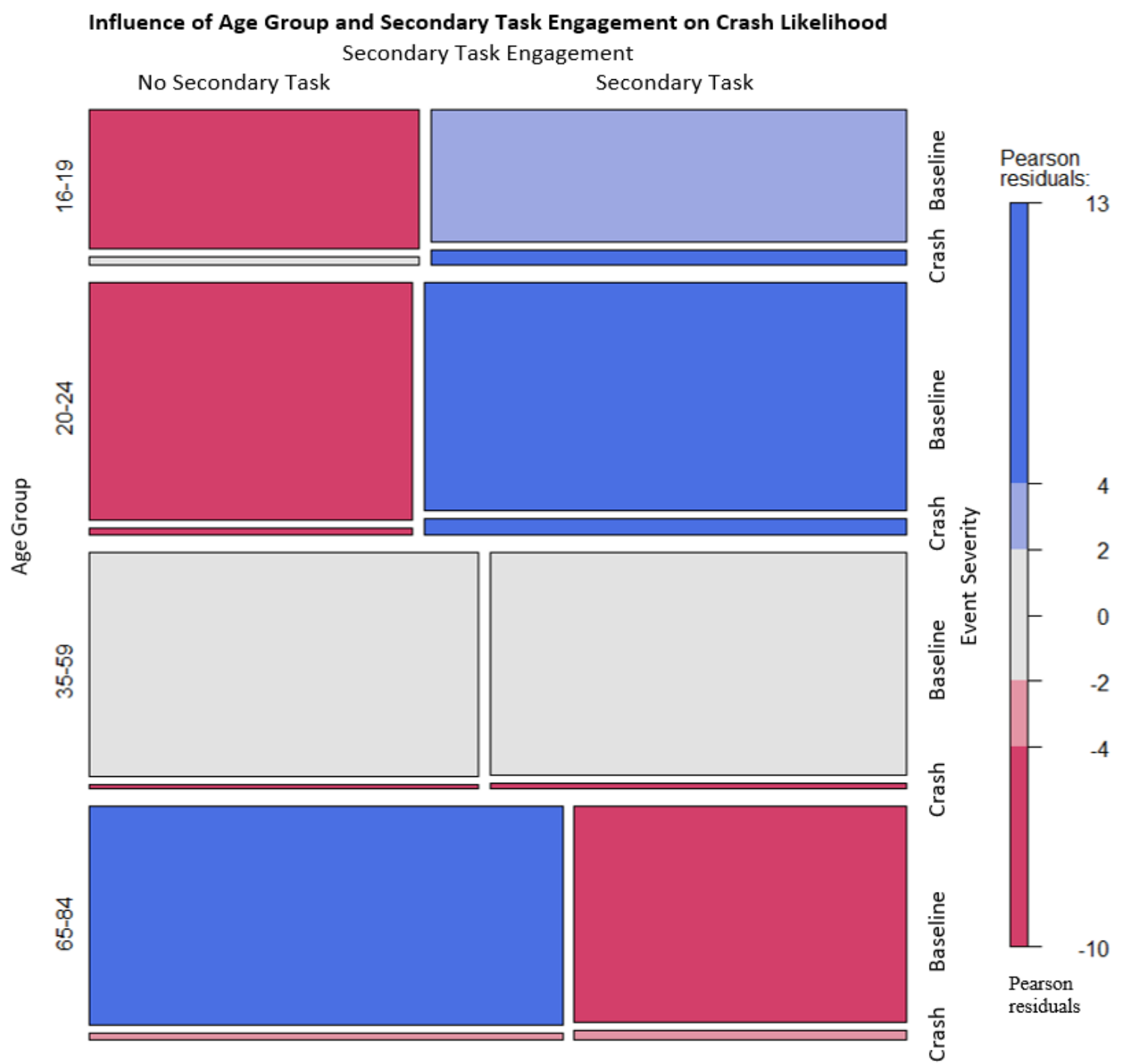

Figure 1. Relationship between age group, distraction, and event severity

\section{REFERENCES}

Dingus TA, et al. (2015) Naturalistic driving study: Technical coordination and quality control (Transportation Research Board, Washington, DC), SHRP 2 Report No. S2-S06-RW-1

Guo, F., Klauer, S. G., Fang, Y., Hankey, J. M., Antin, J. F., Perez, M. A., . . Dingus, T. A. (2017). The effects of age on crash risk associated with driver distraction. International Journal of Epidemiology, 46(1), 258-265. doi:10.1093/ije/dyw234

Hankey, J. M., McClafferty, J. A., \& Perez, M. A. (2016). Description of the SHRP 2 naturalistic database and the crash, near-crash, and baseline data sets. (Transportation Research Board of The National Academies Report). Retrieved from Virginia Tech, Virginia Tech Transportation Institute, Technical Reports website: https://vtechworks.lib.vt.edu/bitstream/handle/10919/70850/SHRP_2_CrashNearCrashBa selineReport_4-25-16.pdf 
Klauer, S. G., Guo, F., Simons-Morton, B. G., Ouimet, M. C., Lee, S. E., \& Dingus, T. A. (2014). Distracted Driving and Risk of Road Crashes among Novice and Experienced Drivers. The New England Journal of Medicine, 370(1), 54-59. doi:10.1056/NEJMsa1204142

Lansdown, T. C., Brook-Carter, N., \& Kersloot, T. (2004). Distraction from multiple in-vehicle secondary tasks: vehicle performance and mental workload implications, Ergonomics, 47(1), 91-104.

Metz, B., Landau, A., \& Just, M. (2014). Frequency of secondary tasks in driving - Results from naturalistic driving data, Safety Science, 68, 195-203.

NHTSA's National Center for Statistics and Analysis. (2018, April). Distracted driving 2016. (Traffic Safety Facts Research Note. Report No. DOT HS 812 517). Washington, DC: National Highway Traffic Safety Administration.

NHTSA. (2018). Traffic Safety Facts 2016 Data: Summary of Motor Vehicle Crashes. (DOT HS 812 580). Washington, DC: US Department of Transportation Retrieved from file://C:/Users/ARGLab1/Downloads/2016\%20Summary\%20of\%20Motor\%20Vehicle\% 20Crashes.pdf.

Owens J. M., Angell, L., Hankey, J. M., Foley, J., \& Ebe, K. (2015). Creation of the Naturalistic Engagement in Secondary Tasks (NEST) distracted driving dataset, Journal of Safety Research, 54, 33-40.

Young, K. \& Regan, M. (2007). Driver distraction: A review of the literature. In: I.J. Faulks, M. Regan, M. Stevenson, J. Brown, A. Porter \& J.D. Irwin (Eds.). Distracted driving. Sydney, NSW: Australasian College of Road Safety. Pages 379-405. 